\title{
High prevalence of oral potentially malignant disorders and risk factors in a semi-urban brazilian city: a population-based cross-sectional study
}

\author{
Allan Vinícius Martins-de-Barros ${ }^{1}$, Ana Maria Ipólito Barros ${ }^{1}$, Caio César Gonçalves Silva ${ }^{2}$, Letícia Francine \\ Silva Ramos ${ }^{3}$, Stefânia Jerônimo Ferreira ${ }^{4}$, Fábio Andrey da Costa Araújo ${ }^{4,5}$, Emanuel Dias de Oliveira e Silva \\ 5, Marianne de Vasconcelos Carvalho ${ }^{4,5}$
}

${ }^{1}$ DDS.Post-GraduationPrograminDentistry/OralMedicineandPathology.SchoolofDentistry,UniversityofPernambuco.Recife,Brazil ${ }^{2}$ DDS, MsC. Post-Graduation Program in Dentistry / Oral and Maxillofacial Surgery. School of Dentistry, University of Pernambuco. Recife, Brazil

${ }^{3}$ Dentistry student. School of Dentistry, University of Pernambuco. Arcoverde, Brazil

${ }^{4}$ DDS, MSc, PhD. Professor. School of Dentistry, University of Pernambuco. Arcoverde, Brazil

${ }^{5}$ DDS, MSc, PhD. Professor. School of Dentistry, University of Pernambuco. Recife, Brazil

Correspondence:

School of Dentistry. University of Pernambuco

Rua Arnóbio Marquês, 310, Santo Amaro, Recife, Pernambuco, Brazil

fabio.andrey@upe.br

Received: $19 / 03 / 2021$

Accepted: 03/05/202
Martins-de-Barros AV, Barros AM, Silva CC, Ramos LF, Ferreira SJ, Araújo FA, et al. High prevalence of oral potentially malignant disorders and risk factors in a semi-urban brazilian city: a population-based cross-sectional study. Med Oral Patol Oral Cir Bucal. 2021 Nov 1;26 (6):e778-85.

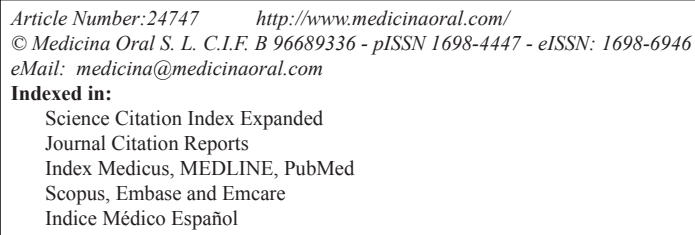

\begin{abstract}
Background: Oral Potentially Malignant Disorders (OPMDs) are defined as lesions with a greater likelihood of progressing to cancer. Population-based studies that evaluate the prevalence of OPMDs are scarce in Brazil. The aim of the present study was to determine the prevalence of OPMDs and associated risk factors in a semi-urban Brazilian population.

Material and Methods: This is a cross-sectional study, whose universe included individuals aged 40 years or older residing in a medium-sized city of northeastern Brazil. Data collection was divided into two steps: interview and oral examination. The outcome variable was the presence of OPMDs. The predictor variables were sociodemographic characteristics and risk habits. The bivariate analysis was performed through chi-square test. The crude prevalence ratios (PR) and its respective $95 \%$ confidence intervals (CI) were calculated. Poisson regression analysis with robust variance was used to calculate adjusted PRs and 95\% CI.

Results: Three hundred fourteen individuals were included in the study. When asked about risk habits, 58.9\% reported being current smokers or ex-smokers and $62.2 \%$ reported being current drinkers or ex-drinkers. The prevalence of OPMDs was $7.6 \%$ and was significantly higher among individuals with black skin color $(p<0.001)$, alcohol users $(p=0.017)$, and individuals with both tobacco and alcohol habits $(p=0.012)$.

Conclusions: Therefore, the population in the present study had a high frequency of risk habits associated with PMDs of the oral cavity.
\end{abstract}

Key words: Oral mucosal lesions, oral cancer, oral potentially malignant disorders, prevalence. 


\section{Introduction}

Oral cancer is considered an important public health problem $(1,2)$. Even with advances in treatment, the five-year survival rate has not increased in decades. Squamous cell carcinoma (SCC) is the most frequent malignant tumor in the oral cavity (3). Oral Potentially Malignant Disorders (OPMDs) are defined as lesions with a greater likelihood of progressing to cancer. In such cases, the tissue exhibits morphological and cellular changes and has a greater tendency to undergo malignant transformation into an SCC compared to normal epithelium (4).

Approximately $95 \%$ of cases of oral cancer occur in individuals older than 40 years of age (3), the majority of whom are men, users of tobacco and alcohol, and have a low socioeconomic status $(5,6)$. Knowledge on the prevalence and profile of the distribution of OPMDs in populations at risk is fundamental to the establishment of public prevention and control policies.

Population-based studies that evaluate the prevalence of OPMDs are scarce in Brazil, especially in the northeastern region of the country. Therefore, the aim of the present study was to determine the prevalence of OPMDs and associated risk factors in individuals 40 years of age or older in a population of a medium-sized semiurban city in the state of Pernambuco, Brazil.

\section{Material and Methods}

\section{- Study design and population}

The present study received approval from the institutional review board of the University of Pernambuco (CAAE: 31240020.7.0000.5191) and was conducted in accordance with the guidelines of the Strengthening the Reporting of Observational Studies in Epidemiology (STROBE) statement for cross-sectional studies.

An observational, cross-sectional, prevalence study was conducted through a population-based epidemiological survey in a medium-sized city in the semiarid region of the state of Pernambuco (northeastern Brazil). Study population was composed of male and female individuals aged 40 years or older residing in the area of coverage of a primary care unit. The exclusion criteria were cognitive, neurological, or psychiatric disorders that impeded answering the questionnaire, the non-localization of individuals at their homes after three attempts at different times of the day, and refusal to participate in any of the steps of the study.

- Sample size calculation and sampling procedure

The sample size was calculated using the highest prevalence for OPMDs (10.54\%) reported in a systematic review conducted by Mello et al. (7) Considering a 99\% confidence interval $(\alpha=0.01), 5 \%$ acceptable margin of error, a design effect of 1.0, and adjustment for a possible $25 \%$ non-response rate, the sample was defined as 314 individuals.
Individuals belonging to the target population (units of analysis) and their respective residential addresses were identified and listed based on e-SUS AB records (electronic primary care records of the Brazilian public healthcare system) kindly granted by the Municipal Secretary of Health. A simple random sampling procedure was employed with the aid of a computer-generated random number table (available at www.random.org) to select the sample.

- Data collection in field

The data collection instrument was a questionnaire with closed-ended questions addressing sociodemographic characteristics (sex, age, and self-reported skin color), risk habits (tobacco and alcohol use), and the presence of oral lesions suspected of being cancer or OPMDs.

Data collection was performed in the respondents' homes by three duly trained researchers who had undergone a calibration exercise for the diagnosis of oral lesions. The collection procedure was divided into two steps: interview and oral examination. The interview consisted of the administration of the questionnaire. The soft tissues of the oral cavity were examined with the aid of an artificial light and mouth mirror. The examination was performed systematically on all anatomic structures of the mouth and oropharynx through a visual inspection and palpation. The researcher recorded the presence/absence of lesions of the oral mucosa exhibiting clinical characteristics consistent with oral cancer, leukoplakia, or erythroplakia based on the criteria established by Warnakulasuriya et al. (8) and noted the clinical characterization of the lesions.

- Database and statistical analysis

The database of the study was created with the aid of the Statistical Package for Social Sciences (SPSS ${ }^{\circledR}$ version 20.0.0). For the bivariate analysis, the variables were categorized dichotomously. The outcome variable was the presence of OPMDs. The predictor variables were sociodemographic characteristics and risk habits. The bivariate analysis was performed through chi-square test. The crude prevalence ratios (PR) and its respective $95 \%$ confidence intervals (CI) were calculated. Poisson regression analysis with robust variance was performed for the calculation of adjusted PRs with $95 \%$ CI. Variables with a p-value $<0.20$ in the bivariate analysis were incorporated into the multivariate model and those with a $p$-value $<0.05$ after the adjustments were considered significantly associated with the outcome.

\section{Results}

Three hundred fourteen individuals were selected to compose the sample of the present study. Seven were not found at their homes after three attempts and three declined to participate in the study. Thus, 304 individuals completed all steps of the data collection and com- 
posed the final sample (Fig. 1). The sociodemographic characteristics of the participants are displayed in Table 1. Age ranged from 40 to 94 years (mean: $62.33 \pm 13.57$ years). Women (69.4\%) and individuals with brown skin color $(62.2 \%)$ accounted for the majority of the sample. When asked about risk habits, 58.9\% reported being current smokers or ex-smokers and $62.2 \%$ reported being current drinkers or ex-drinkers. Only 62 individuals $(20.4 \%)$ never used tobacco or alcoholic beverages. Among the smokers and ex-smokers, the largest portion $(49.2 \%)$ reported smoking less than 10 cigarettes/ day and $7.8 \%$ reported smoking more than 40 cigarettes/day. The type of cigarette most frequently used was of paper $(86 \%)$. Regarding the alcohol intake pattern, 97 individuals reported the use of more than one type of beverage. Beer was the most frequent (69.9\% of drinkers), whereas 27 of those who drank reported drinking beer, wine, cachaça (distilled spirit made from fermented sugarcane juice), and other distilled spirits. The quantity of drinks per day that each individual consumed was also investigated: $63 \%$ reported occasional use and $14.3 \%$ reported having or having had more than six drinks per day. Total of $47.8 \%$ of ex-smokers and $41.8 \%$ of ex-drinkers reported having quit the habit more than 20 years earlier. The absolute and relative frequencies of smoking and drinking patterns are displayed in Table 1.

Smoking experience (smokers and ex-smokers) was significantly associated with skin color $(p=0.002)$. Alcohol experience (drinkers and ex-drinkers) was significantly associated with both age $(p<0.001)$ and sex $(p$ $<0.001$ ) (Table 2).

The physical examinations of the oral cavity revealed lesions suspected of being OPMDs in 23 individuals (7.6\%). Seventeen lesions were characterized as leukoplakia, five were characterized as erythroplakia, and only one was characterized as erythroleukoplakia. No lesions suggestive of SCC were identified.

The crude PRs for OPMDs and p-values from the chisquared test are displayed in Table 3 . In the bivariate analysis, the presence of OPMDs was significantly associated with skin color $(p=0.001)$ and alcohol use ( $p$ $=0.047$ ).

The adjusted PRs and respective 95\% CIs are displayed in Table 4. The prevalence of OPMDs was significantly higher among individuals with black skin color (PR: 4.783; 95\% CI: 2.327 to 9.831; $p<0.001$ ), alcohol users (PR: 3.207 ; 95\% CI: 1.229 to $8.369 ; p=0.017$ ), and individuals with both tobacco and alcohol habits (PR: 4.143; 95\% CI: 1.369 to $12.541 ; p=0.012$ ).

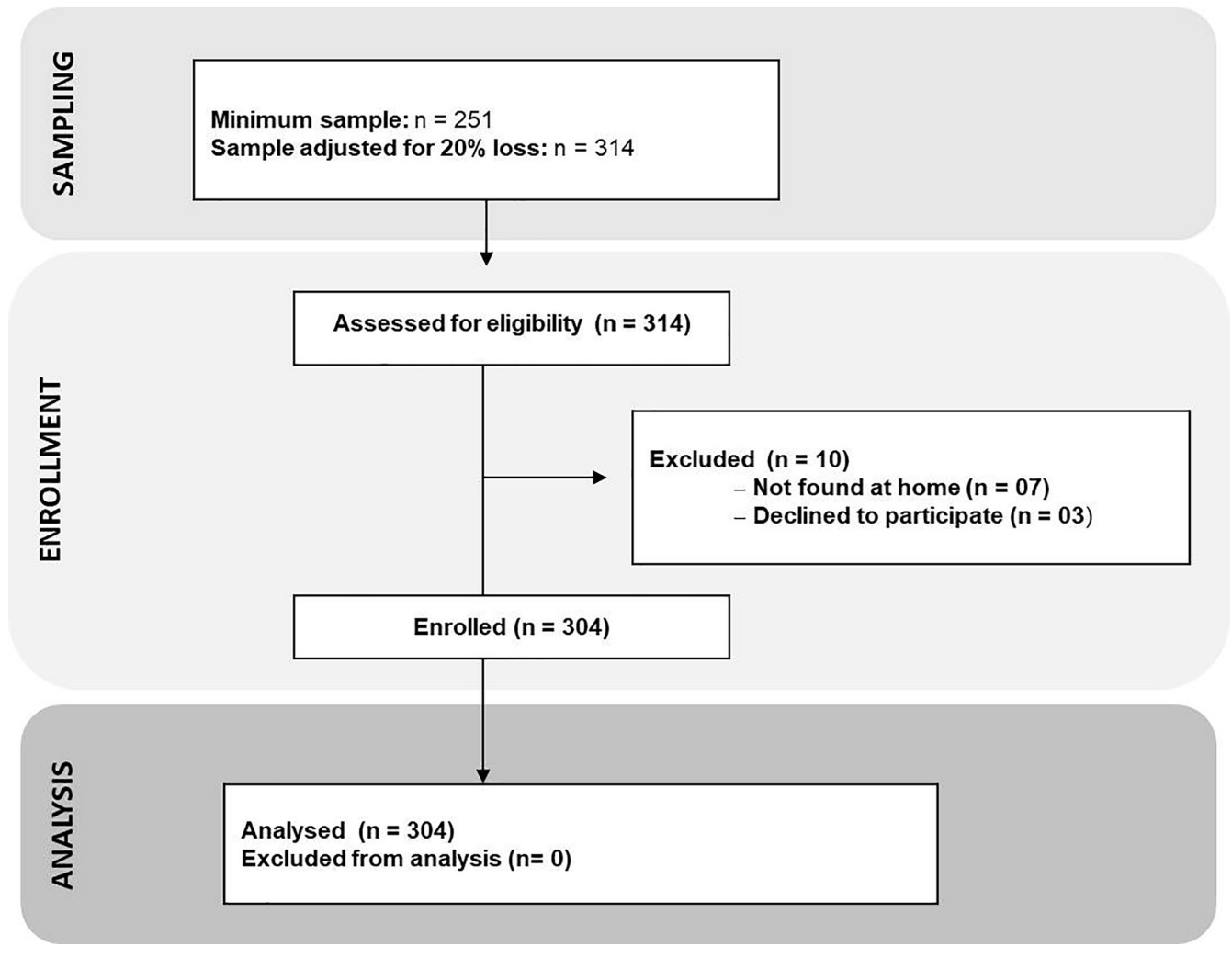

Fig. 1: Participant Flowchart. 
Table 1: Sociodemographic charactesitics and frequency of risk habits among the participants of the study.

\begin{tabular}{|c|c|c|c|c|c|}
\hline \multicolumn{6}{|c|}{ Sociodemographic variable $s$} \\
\hline \multicolumn{3}{|l|}{ Age } & \multicolumn{3}{|l|}{ years } \\
\hline \multicolumn{3}{|l|}{ Age range } & \multicolumn{3}{|l|}{$40-94$} \\
\hline \multicolumn{3}{|l|}{ Mean \pm SD } & \multicolumn{3}{|l|}{$62.33 \pm 13.57$} \\
\hline \multicolumn{3}{|l|}{ Sex } & $\mathbf{n}$ & \multicolumn{2}{|c|}{$\%$} \\
\hline \multicolumn{3}{|l|}{ Women } & 211 & \multicolumn{2}{|c|}{69.4} \\
\hline \multicolumn{3}{|l|}{ Men } & 93 & \multicolumn{2}{|c|}{30.6} \\
\hline \multicolumn{3}{|l|}{ Skin color } & $\mathbf{n}$ & \multicolumn{2}{|c|}{$\%$} \\
\hline \multicolumn{3}{|l|}{ Brown } & 189 & \multicolumn{2}{|c|}{62.2} \\
\hline \multicolumn{3}{|l|}{ Black } & 58 & & \\
\hline White & & & 54 & & 4 \\
\hline Others & & & 01 & & \\
\hline Total & & & 304 & & 0 \\
\hline & & Risk & habits & & \\
\hline Smoker & $\mathbf{n}$ & $\%$ & Alcohol drinker & $\mathbf{n}$ & $\%$ \\
\hline Yes & 66 & 21.7 & Yes & 91 & 29.9 \\
\hline No & 238 & 78.3 & No & 213 & 70.1 \\
\hline How long do you smoke? & $\mathbf{n}$ & $\%$ & How long do you drink? & $\mathbf{n}$ & $\%$ \\
\hline$<5$ years & 0 & 0.0 & $<5$ years & 10 & 10.9 \\
\hline 6 a 10 years & 5 & 7.6 & 6 a 10 years & 04 & 4.4 \\
\hline 11 a 20 years & 5 & 7.6 & 11 a 20 years & 15 & 16.5 \\
\hline$>20$ years & 56 & 84.8 & $>20$ years & 62 & 68.2 \\
\hline Ex-smoker & $\bar{n}$ & $\%$ & Ex-alcohol drinker & $\mathbf{n}$ & $\%$ \\
\hline Yes & 113 & 37.2 & Yes & 98 & 32.2 \\
\hline No & 191 & 62.8 & No & 206 & 67.8 \\
\hline How long did you smoke? & $\mathbf{n}$ & $\%$ & How long did you drink? & $\mathbf{n}$ & $\%$ \\
\hline$<5$ years & 20 & 17.7 & $<5$ years & 25 & 25.5 \\
\hline 6 a 10 years & 19 & 16.8 & 6 a 10 years & 13 & 13.2 \\
\hline 11 a 20 years & 17 & 15.0 & 11 a 20 years & 12 & 12.3 \\
\hline$>20$ years & 57 & 50.5 & $>20$ years & 48 & 49.0 \\
\hline How long have you stopped smoking? & $\mathbf{n}$ & $\%$ & How long have you stopped drinking? & $\mathbf{n}$ & $\%$ \\
\hline$<5$ years & 26 & 23.0 & $<5$ years & 29 & 29.6 \\
\hline 6 a 10 years & 11 & 9.7 & 6 a 10 years & 17 & 17.4 \\
\hline 11 a 20 years & 22 & 19.5 & 11 a 20 years & 11 & 11.2 \\
\hline$>20$ years & 54 & 47.8 & $>20$ years & 41 & 41.8 \\
\hline Number of cigarettes/day & $\mathbf{n}$ & $\%$ & Number of drinks/day & $\mathbf{n}$ & $\%$ \\
\hline$<10$ cigarettes/day & 88 & 49.2 & $<2$ drinks/day & 21 & 11.1 \\
\hline 10 a 20 cigarettes/day & 58 & 32.4 & 2 a 4 drinks/day & 15 & 7.9 \\
\hline 21 a 40 cigarettes/day & 19 & 10.6 & 5 a 6 drinks/day & 07 & 3.7 \\
\hline$>40$ cigarettes/day & 14 & 7.8 & $>6$ drinks/day & 27 & 14.3 \\
\hline & & & Occasional use & 119 & 63.0 \\
\hline Type of cigarette & n & $\%$ & Type of alcoholic drink & n & $\%$ \\
\hline Paper cigarette & 154 & 86.0 & Beer & 132 & 69.9 \\
\hline Smoking pipe & 12 & 6.7 & Wine & 59 & 31.2 \\
\hline Straw cigarette & 41 & 22.9 & Cachaça & 98 & 51.8 \\
\hline Cigar & 01 & 0.6 & Other distilled & 69 & 36.5 \\
\hline
\end{tabular}

SD, standard deviation. 
Table 2: Relationship between smoking and alcohol experience and sociodemographic characteristics among the participants of the study.

\begin{tabular}{|c|c|c|c|c|c|c|}
\hline \multicolumn{7}{|c|}{ Smoking experience } \\
\hline & $\begin{array}{c}\text { Yes } \\
\text { n (\%) }\end{array}$ & $\begin{array}{c}\text { No } \\
\text { n (\%) }\end{array}$ & $\mathbf{X}^{2}$ & PR & CI 95\% & $p$-value \\
\hline \multicolumn{7}{|l|}{ Age } \\
\hline $40-61$ years & $94(58.4 \%)$ & $67(41.6 \%)$ & \multirow{2}{*}{0.35} & \multirow{2}{*}{0.957} & \multirow{2}{*}{$0.606-1.513$} & \multirow{2}{*}{0.472} \\
\hline$>61$ years & $85(59.4 \%)$ & $58(40.6 \%)$ & & & & \\
\hline \multicolumn{7}{|l|}{ Sex } \\
\hline Men & $59(63.4 \%)$ & $34(36.6 \%)$ & \multirow{2}{*}{1.15} & \multirow{2}{*}{1.230} & \multirow{2}{*}{$0.796-2.175$} & \multirow{2}{*}{0.172} \\
\hline Women & $120(56.9 \%)$ & $91(43.1 \%)$ & & & & \\
\hline \multicolumn{7}{|l|}{ Skin color } \\
\hline Black & $44(75.9 \%)$ & $14(24.1 \%)$ & \multirow{2}{*}{8.53} & \multirow{2}{*}{2.584} & \multirow{2}{*}{$1.347-4.959$} & \multirow{2}{*}{$0.002 *$} \\
\hline White, brown and others & $135(54.9 \%)$ & $111(45.1 \%)$ & & & & \\
\hline \multicolumn{7}{|c|}{ Alcohol experience } \\
\hline & $\begin{array}{c}\text { Yes } \\
\text { n (\%) }\end{array}$ & $\begin{array}{c}\text { No } \\
\text { n (\%) }\end{array}$ & $\mathbf{X}^{2}$ & PR & CI 95\% & $p$-value \\
\hline \multicolumn{7}{|l|}{ Age } \\
\hline $40-61$ years & $119(73.9 \%)$ & $42(26.1 \%)$ & \multirow{2}{*}{20.06} & \multirow{2}{*}{2.955} & \multirow{2}{*}{$1.827-4.779$} & \multirow{2}{*}{$<0.001 *$} \\
\hline$>61$ years & $70(49.0 \%)$ & $73(51.0 \%)$ & & & & \\
\hline \multicolumn{7}{|l|}{ Sex } \\
\hline Men & $78(83.9 \%)$ & $15(16.1 \%)$ & \multirow{2}{*}{26.82} & \multirow{2}{*}{4.685} & \multirow{2}{*}{$2.532-8.666$} & \multirow{2}{*}{$<0.001 *$} \\
\hline Women & $111(52.6 \%)$ & $100(47.4 \%)$ & & & & \\
\hline \multicolumn{7}{|l|}{ Skin color } \\
\hline Black & $40(69.0 \%)$ & $18(31.0 \%)$ & \multirow{2}{*}{1.40} & \multirow{2}{*}{1.447} & \multirow{2}{*}{$0.784-2.668$} & \multirow{2}{*}{0.150} \\
\hline White, brown and others & $149(60.6 \%)$ & $97(39.4 \%)$ & & & & \\
\hline
\end{tabular}

$\mathrm{X}^{2,}$ Chi-square value. PR, prevalence ratio. CI, confidence interval. * $p<0,05$

Table 3: Relationship between Oral Potentially Malignant Disorders and sociodemographic characteristics and risk habits among the participants of the study.

\begin{tabular}{|c|c|c|c|c|c|c|}
\hline & \multicolumn{6}{|c|}{$\begin{array}{c}\text { Presence of Oral Potentially } \\
\text { Malignant Disorders }\end{array}$} \\
\hline & $\begin{array}{c}\text { Yes } \\
\text { n (\%) }\end{array}$ & $\begin{array}{c}\text { No } \\
\text { n (\%) }\end{array}$ & $\mathbf{X}^{2}$ & $\mathbf{P R}$ & CI 95\% & $p$-value \\
\hline \multicolumn{7}{|l|}{ Age } \\
\hline $40-61$ years & $11(6.8 \%)$ & $150(93.2 \%)$ & \multirow{2}{*}{0.26} & \multirow{2}{*}{0.801} & \multirow{2}{*}{$0.342-1.875$} & \multirow{2}{*}{0.383} \\
\hline$>61$ years & $12(8.4 \%)$ & $131(91.6 \%)$ & & & & \\
\hline \multicolumn{7}{|l|}{ Sex } \\
\hline Men & $8(8.6 \%)$ & $85(91.4 \%)$ & \multirow{2}{*}{0.20} & \multirow{2}{*}{1.230} & \multirow{2}{*}{$0.502-3.010$} & \multirow{2}{*}{0.404} \\
\hline Women & $15(7.1 \%)$ & $196(92.9 \%)$ & & & & \\
\hline \multicolumn{7}{|l|}{ Skin color } \\
\hline Black & $11(19.0 \%)$ & $47(81.0 \%)$ & \multirow{2}{*}{13.31} & \multirow{2}{*}{4.546} & \multirow{2}{*}{$1.900-10.960$} & \multirow{2}{*}{ 0.001* } \\
\hline White, brown and others & $12(4.9 \%)$ & $234(95.1 \%)$ & & & & \\
\hline \multicolumn{7}{|l|}{ Smoker } \\
\hline Yes & $8(12.1 \%)$ & $58(87.9 \%)$ & \multirow{2}{*}{2.50} & \multirow{2}{*}{2.051} & \multirow{2}{*}{$0.829-5.071$} & \multirow{2}{*}{0.097} \\
\hline No & $15(6.3 \%)$ & $223(93.7 \%)$ & & & & \\
\hline \multicolumn{7}{|l|}{ Alcohol drinker } \\
\hline Yes & $11(12.1 \%)$ & $80(87.9 \%)$ & \multirow{2}{*}{3.79} & \multirow{2}{*}{2.303} & \multirow{2}{*}{$0.976-5.433$} & \multirow{2}{*}{$0.047 *$} \\
\hline No & $12(5.6 \%)$ & $201(94.4 \%)$ & & & & \\
\hline Total & $23(7,6 \%)$ & $281(92.4 \%)$ & - & - & - & - \\
\hline
\end{tabular}


Table 4: Adjusted Prevalence Ratios and its respective 95\% Confidence Interval for the association between the presence of Oral Potentially Malignant Disorders and skin color, smoking and alcohol drinking habits.

\begin{tabular}{|l|c|c|}
\hline & Adjusted PR (CI 95\%) & $p$-value \\
\hline Skin color & \multicolumn{2}{|c|}{} \\
\hline Black & $4.783(2.327-9.831)$ & $<\mathbf{0 . 0 0 1 *}$ \\
\hline White, brown and others & 1.00 & - \\
\hline Risk habits & & \\
\hline Smoker and alcohol drinker & $4.143(1.369-12.541)$ & $\mathbf{0 . 0 1 2 *}$ \\
\hline Non-smoker and alcohol drinker & $3.207(1.229-8.369)$ & $\mathbf{0 . 0 1 7}$ \\
\hline Smoker and non-alcohol drinker & $1.971(0.695-5.590)$ & 0.202 \\
\hline Non-smoker and non-alcohol drinker & 1.00 & - \\
\hline
\end{tabular}

PR, prevalence ratio. CI, confidence interval. ${ }^{*} p<0,05$

\section{Discussion}

SCCs and OPMDs are pathological changes originating in the lining tissue of the oral cavity and are directly associated with demographic characteristics, such as age, as well as risk habits, such as smoking and alcohol intake (9). As the prevalence of these lesions and associated risk factors varies significantly in different populations and geographic regions, knowledge on the local-regional epidemiological status is fundamental to the establishment of prevention measures and the early diagnosis of these conditions.

The World Health Organization strongly recommends population-based epidemiological surveys to determine the prevalence of oral lesions related to cancer (10). However, few studies with this methodological design are found in the scientific literature. In Brazil, prevalence data regarding malignant and potentially malignant lesions of the oral cavity are mainly from studies conducted at institutions or healthcare establishments with non-random samples, which substantially increases the risk of bias and compromises the external validity of the findings.

The pooled global prevalence of OPMDs calculated in a meta-analysis of a systematic review conducted by Mello et al. (7) was $4.47 \%$. When only considering leukoplakia and erythroplakia, the prevalence was $4.28 \%$ (9). In the present study, a much higher rate of PMDs in the oral cavity clinically characterized as leukoplakia, erythroplakia, and erythroleukoplakia was found in the population studied (7.6\%). Population-based crosssectional studies conducted in Brazil, such as the investigation by Ferreira et al. (10), report a much lower prevalence of oral leukoplakia (2.3\%) and erythroplakias $(0.3 \%)$ in rural workers. In contrast, Pivovar et al. (11) found a nearly $25 \%$ rate of leukoplakia in a population classified as having a high risk of oral cancer. The considerable variability in the prevalence of these disorders may be related to sociodemographic characteristics and the frequency of cultural practices considered risk habits in different populations.
Smoking and alcohol use are well-established etiological factors for OPMDs and play an equally important role in the progression of these lesions $(9,12)$. In the multivariate model of the present study, the prevalence of OPMDs was significantly higher among individuals who used tobacco or alcoholic beverages. Moreover, due to the synergistic effect, the prevalence was 4.14fold higher among those who reported the concomitant use of these two substances.

Although the prevalence of these habits has diminished in Brazil in recent years, there is a marked inequality in the reach and effectiveness of prevention measures in different socioeconomic strata $(13,14,15)$. Populations who live in the interior of the state are expected to behave differently compared to those who live in large metropolises in terms of risk factors associated with OPMDs and oral cancer. The rates of smoking and alcohol use in the present study are higher than the average for Brazil as a whole and for the northeastern region in particular $(13,14)$. This finding is compatible to data reported by Amarasinghe et al. (16) for a rural population in the interior or Sri Lanka. Thus, regional differences in the prevalence of OPMDs seem to accompany those of tobacco and alcohol use.

There is no consensus in the literature on the association between sex and oral OPMDs, as the distribution of such disorders is directly influenced by socioeconomic and cultural aspects (7). Although some clinical types of OPMDs, such as actinic cheilitis, are more prevalent among men (10), no significant difference between the sexes was found regarding the prevalence of OPMDs in the present study. This may partially be explained by the greater similarity in the social behavioral patterns of men and women in instate populations regarding risk habits, especially smoking. Indeed, no significant difference in smoking experience was found between the sexes.

Few studies have analyzed the distribution of OPMDs in different ethnic groups. In a systematic review conducted by Mello et al. (7), the authors found a greater 
frequency of OPMDs in "light skin" individuals. In contrast, the prevalence was 4.78 -fold greater among black skin individuals in the present investigation. This difference is probably not due to biological factors, as the quantity and distribution of melanin in tissues is neither a risk factor nor protection factor for the development of OPMDs, mainly because these are lesions for which ultraviolet solar radiation is not a determinant etiological factor. The greater prevalence in black individuals seems to be related to the marked social inequality and greater difficulty in gaining access to services and information. Kravietz et al. (17) highlighted important racial inequality regarding access to information and screening services for head and neck cancer in the United States. Such inequality tends to be greater in municipalities distant from metropolises, which contributes to an increased risk of oral cancer and OPMDs in these populations.

Although lesions located in the mouth are accessible to a visual inspection and palpation (18), the diagnosis is generally performed when such lesions are already in late stages (3). Although there is little current evidence on the effectiveness of screening programs for oral cancer (19), early detection of OPMDs in stages prior to a possible malignant transformation has the potential to reduce morbidity and mortality rates related to these conditions, especially in individuals exposed to risk factors $(7,18)$. However, there is a lack of decentralized programs for the early identification of oral lesions in instate regions (10). In this regard, the data of the present study can assist in guiding such programs to populations at greater risk.

The present study has limitations that should be considered. The cross-sectional design does not enable the establishment of a cause-and-effect relationship between exposure and outcome in the analysis of associations between variables. The lack of histopathological data impedes a more in-depth analysis of morphological changes and the potential for the progression to malignancy in the lesions clinically diagnosed as OPMDs. Moreover, the lack of methodological standardization among prevalence studies in the literature regarding sampling procedures and the diagnosis of lesions compromises the uniformity of the data and hinders the comparison of the results.

In conclusion, the population in the present study had a high prevalence of OPMDs and associated risk habits. The majority of lesions were clinically characterized as leukoplakia. The prevalence of OPMDs was higher among individuals with black skin color and those who had the concomitant habits of smoking and alcohol use. These findings underscore the importance of intensifying policies aimed at primary prevention and the early diagnosis of OPMDs and oral cancer with a focus on the dissemination of information and the control of risk habits, especially in more vulnerable geographic areas and socioeconomic strata, as lifestyle and living conditions in rural and semi-urban areas differ considerably from those found in large urban centers.

\section{References}

1. Jemal A, Siegel R, Xu J, Ward E. Cancer Statistics, 2010. CA Cancer J Clin. 2011;61:133-4.

2. Abed H, Reilly D, Burke M, Daly B. Patients with head and neck cancers' oral health knowledge, oral health-related quality of life, oral health status, and adherence to advice on discharge to primary dental care: A prospective observational study. Spec Care Dentist. 2019;39:593-602.

3. Mortazavi H, Baharvand M, Mehdipour M. Oral Potentially Malignant Disorders: An Overview of More than 20 Entities. J Dent Res Dent Clin Dent Prospects. 2014;8:6-14.

4. Ganesh D, Sreenivasan P, Öhman J, Wallström M, Braz-Silva PH, Giglio D, et al. Potentially Malignant Oral Disorders and Cancer Transformation. Anticancer Res. 2018;38:3223-9.

5. Shrestha AD, Vedsted P, Kallestrup P, Neupane D. Prevalence and incidence of oral cancer in low- and middle-income countries: A scoping review. Eur J Cancer Care (Engl). 2020;29:e13207.

6. Wong TSC, Wiesenfeld D. Oral Cancer. Aust Dent J. 2018;63:S91-9. 7. Mello FW, Miguel AFP, Dutra KL, Porporatti AL, Warnakulasuriya S, Guerra ENS, et al. Prevalence of oral potentially malignant disorders: a systematic review and meta-analysis. J Oral Pathol Med. 2018;47:633-40.

8. Warnakulasuriya S, Johnson NW, van der Waal I. Nomenclature and classification of potentially malignant disorders of the oral mucosa. J Oral Pathol Med. 2007;36:575-80.

9. Kadashetti V, Shivakumar KM, Chaudhary M, Patil S, Gawande $\mathrm{M}$, Hande A. Influence of risk factors on patientes suferring from potentially malignant disorders and oral cancer: A case-control study. J Oral and Maxillofac Pathol. 2012;21:455-6.

10. Ferreira AM, de Souza Lucena EE, de Oliveira TC, da Silveira EJD, de Oliveira PT, de Lima KC. Prevalence and factors associated with oral potentially malignant disorders in Brazil's rural workers. Oral Diseases. 2016;22:536-42.

11. Pivovar A, Santos ZFDG, Torres-Pereira CC. Oral cancer screening for high-risk individuals in the primary healthcare setting using an active approach. J Oral Pathol Med. 2017;46:786-91.

12. Speight PM, Khurram SA, Kujan O. Oral potentially malignant disorders: risk of progression to malignancy. Oral Surg Oral Med Oral Pathol Oral Radiol. 2018;125:612-27.

13. Barros ADJ, Cascaes AM, Wehrmeister FC, Martínez-Mesa J, Menezes AMB. Tobacco smoking in Brazil: regional inequalities and prevalence according to occupational characteristics. Cien Saude Colet. 2011;16:3707-16.

14. Ferreira LN, Sales ZN, Casotti CA, Bispo Júnior JP, Braga Júnior ACR. Alcohol consumption and associated factors in a city in Northeast Brazil. Cad Saúde Pública. 2011;27:1473-86.

15. Pinto MT, Pichon-Riviere A, Bardach A. The burden of smokingrelated diseases in Brazil: mortality, morbidity and costs. Cad Saúde Pública. 2015;31:1-14.

16. Amarasinghe AAHK, Usgodaarachchi US, Johnson NW, Warnakulasuriya S. High Prevalence of Lifestyle Factors Attributable for Oral Cancer, and of Oral Potentially Malignant Disorders in Rural Sri Lanka. Asian Pac J Cancer Prev. 2018;19:2485-92.

17. Kravietz A, Angara P, Le M, Sargi Z. Disparities in Screening for Head and Neck Cancer: Evidence from the NHANES, 2011-2014. Otolaryngol Head Neck Surg. 2018;159:683-91.

18. Villa A, Gohel A. Oral potentially malignant disorders in a large dental population. J Appl Oral Sci. 2014;22:473-6.

19. Brocklehurst PR, Speight PM. Screening for mouth cancer: the pros and cons of a national programme. Br Dental J. 2018;225:815-9. 


\section{Funding}

None declared.

\section{Conflict of interest}

The authors have no conflict of interest to declare.

\section{Ethics}

This study was approved by the Committee of Ethics of the University of Pernambuco (CAAE: 31240020.7.0000.5191, all procedures performed in this study were in accordance with the ethical standards of the institutional and national research committee and with the Helsinki declaration and its later amendments or comparable ethical standards.

Informed consent was obtained from all individual participants included in the study.

\section{Authors contributions}

AVMB. The author contributed to concept and design of the study; definition of intellectual content; literature search; data acquisition; data analysis; manuscript preparation; manuscript editing; and manuscript review.

AMIB. The author contributed to literature search; data acquisition; data and statistical analysis; manuscript preparation; manuscript editing; and manuscript review.

CCGS. The author contributed to literature search; data and statistical analysis; manuscript preparation; and manuscript review.

LFSR. The author contributed to study design; data acquisition; data analysis; manuscript preparation; and manuscript review.

SJF. The author contributed to definition of intellectual content; literature search; data analysis; manuscript preparation; manuscript editing; and manuscript review.

FACA. The author contributed to literature search; data acquisition; data analysis; manuscript preparation; manuscript editing; and manuscript review.

EDOS. The author contributed to definition of intellectual content and study design; literature search; manuscript preparation; manuscript editing; and manuscript review.

MVC. The author was the coordinator of the study and contributed to its concept and design; definition of intellectual content; literature search; data acquisition; manuscript preparation; manuscript editing; and manuscript review. 